\title{
Viscosity approximation methods for the multiple-set split equality common fixed-point problems of demicontractive mappings
}

\author{
Yaqin Wang*, Xiaoli Fang
}

Department of Mathematics, Shaoxing University, Shaoxing 312000, China.

Communicated by $\mathrm{X}$. Qin

\begin{abstract}
In this paper, we consider a new parallel algorithm combining viscosity approximation methods to approximate the multiple-set split common fixed point problem governed by demicontractive mappings, and get the generated sequence converges strongly to a solution of this problem. The results obtained in this paper generalize and improve the recent ones announced by many others. (C)2017 All rights reserved.
\end{abstract}

Keywords: Multiple-set split equality common fixed-point problem, demicontractive mapping, strong convergence. 2010 MSC: 47H09, 47H10, 47J05, 54H25.

\section{Introduction}

Let $\mathrm{H}$ be a real Hilbert space with inner product $\langle\cdot, \cdot\rangle$ and norm $\|\cdot\|$. The convex feasibility problem (CFP) is formulated as follows.

$$
\text { If } \cap_{i=1}^{n} C_{i} \neq \emptyset \text {, find a point } x^{*} \in \cap_{i=1}^{n} C_{i},
$$

where $n \geqslant 1$ is an integer, and each $C_{i}$ is a nonempty closed convex subset of $H$. It has been proved that the CFP has received so much attention due to its extensive applications in many applied disciplines as diverse as approximation theory, image recovery and signal processing, and so on. A complete and exhaustive study on algorithms and applications for solving the CFP can be found in $[3,5,12,13]$. As a special case of the CFP, the split feasibility problem can be stated as follows.

The split feasibility problem (SFP) in finite-dimensional Hilbert spaces was first introduced by Censor and Elfving [2]. The SFP is to find

$$
x^{*} \in C \text { such that } A x^{*} \in Q \text {, }
$$

where $C$ and $Q$ are nonempty closed convex subsets of the Hilbert spaces $H_{1}$ and $H_{2}$, respectively, $A$ : $\mathrm{H}_{1} \rightarrow \mathrm{H}_{2}$ is a bounded linear operator. It has been found that the SFP (1.1) can be used in many areas such

\footnotetext{
*Corresponding author

Email addresses: wangyaqin0579@126.com (Yaqin Wang), fx10418@126.com (Xiaoli Fang)
} 
as image restoration, computer tomograph, and radiation therapy treatment planning. Some methods have been proposed to solve split feasibility problems; see, for instance, $[1,17,18,19]$.

Note that if the SFP (1.1) is consistent, it is no hard to see that $x^{*}$ solves the SFP (1.1) if and only if it solves the fixed point equation

$$
x^{*}=P_{C}\left(I-\gamma A^{*}\left(I-P_{Q}\right) A\right) x^{*},
$$

where $P_{C}$ and $P_{Q}$ are the metric projections from $H_{1}$ onto $C$ and from $H_{2}$ onto $Q$, respectively, $\gamma$ is a positive constant and $A^{*}$ denotes the adjoint of $A$ (see [15, Proposition 3.2] for the details). This implies that the SFP (1.1) can be solved by using fixed point algorithms.

In 2013, Moudafi and Al-Shemas [11] introduced the following new split feasibility problem, which is called the split equality fixed point problem (SEFP). Let $\mathrm{H}_{1}, \mathrm{H}_{2}, \mathrm{H}_{3}$ be real Hilbert spaces, let $A: \mathrm{H}_{1} \rightarrow$ $\mathrm{H}_{3}$, B : $\mathrm{H}_{2} \rightarrow \mathrm{H}_{3}$ be two bounded linear operators, let $\mathrm{U}: \mathrm{H}_{1} \rightarrow \mathrm{H}_{1}$ and $\mathrm{T}: \mathrm{H}_{2} \rightarrow \mathrm{H}_{2}$ be two firmly quasi-nonexpansive mappings. The SEFP in [11] is to

$$
\text { find } x^{*} \in F(U), y^{*} \in F(T) \text { such that } A x^{*}=B y^{*} \text {. }
$$

The interest is to cover many situations, for instance, in decomposition methods for PDF's, applications in game theory and in intensity-modulated radiation therapy (IMRT).

For solving the SEFP (1.2), Moudafi and Al-Shemas [11] introduced the following simultaneous iterative method:

$$
\left\{\begin{array}{l}
x_{k+1}=u\left(x_{k}-\gamma_{k} A^{*}\left(A x_{k}-B y_{k}\right)\right) \\
y_{k+1}=T\left(y_{k}+\gamma_{k} B^{*}\left(A x_{k}-B y_{k}\right)\right)
\end{array}\right.
$$

for firmly quasi-nonexpansive mappings $U$ and $T$, where $\gamma_{k} \in\left(\epsilon, \frac{2}{\lambda_{A}+\lambda_{B}}-\epsilon\right), \lambda_{A}, \lambda_{B}$ stand for the spectral radiuses of $A^{*} A$ and $B^{*} B$, respectively.

In 2016, Zhao and Wang [21] proposed the following viscosity iterative algorithm for solving the SEFP (1.2):

$$
\left\{\begin{array}{l}
u_{k}=x_{k}-\gamma_{k} A^{*}\left(A x_{k}-B y_{k}\right) \\
x_{k+1}=\alpha_{k} f_{1}\left(x_{k}\right)+\left(1-\alpha_{k}\right)\left(\left(1-w_{k}\right) u_{k}+w_{k} u\left(u_{k}\right)\right) \\
v_{k}=y_{k}+\gamma_{k} B^{*}\left(A x_{k}-B y_{k}\right) \\
y_{k+1}=\alpha_{k} f_{2}\left(y_{k}\right)+\left(1-\alpha_{k}\right)\left(\left(1-w_{k}\right) v_{k}+w_{k} T\left(v_{k}\right)\right)
\end{array}\right.
$$

where $f_{1}: H_{1} \rightarrow H_{1}$ and $f_{2}: H_{2} \rightarrow H_{2}$ are two contractions, $U: H_{1} \rightarrow H_{1}$ and $T: H_{2} \rightarrow H_{2}$ are quasi-nonexpansive. They proved a strong convergence result of the algorithm (1.3) in Hilbert spaces.

Recently, the multiple-set split equality common fixed-point problem (MSECFP) of quasi-nonexpansive mappings studied by Zhao and Wang [20] is to

$$
\text { find } x^{*} \in \cap_{i=1}^{p} F\left(U_{i}\right), y^{*} \in \cap_{j=1}^{q} F\left(T_{j}\right) \text { such that } A x^{*}=B y^{*},
$$

where $p, q \geqslant 1$ are integers. They introduced two mixed cyclic and parallel iterative algorithms for solving the MSECFP (1.4) of quasi-nonexpansive mappings and proved the weak convergence of these two algorithms.

Inspired and motivated by the works mentioned above, we consider a new viscosity iterative algorithm for the MSECFP (1.4) of demicontractive mappings which are generalization of quasi-nonexpansive mappings in Hilbert spaces. Under some mild assumptions we obtain some strong convergence results for solving the MSECFP (1.4) and the SEFP (1.2).

\section{Preliminaries}

Throughout this paper, we always assume that $\mathrm{H}_{1}, \mathrm{H}_{2}, \mathrm{H}_{3}$ are real Hilbert spaces and let $\mathbb{N}$ and $\mathbb{R}$ be the set of positive integers and real numbers, respectively. We use $\rightarrow$ and $\rightarrow$ to denote strong and weak convergence, respectively, and $F(T)$ denotes the set of the fixed points of a mapping $T$. We use 
$\omega_{w}\left(x_{k}\right)=\left\{x: \exists x_{k_{j}} \rightarrow x\right\}$ to stand for the weak $\omega$-limit set of $\left\{x_{k}\right\}$ and use $\Gamma$ to stand for the solution set of the MSECFP (1.4).

Let $\mathrm{C}$ be a nonempty closed convex subset of a Hilbert space $H$. The metric (or nearest point) projection $P_{C}$ from $H$ onto $C$ is defined as follows: Given $x \in H$, the unique point $P_{C} x \in C$ satisfies the property

$$
\left\|x-P_{C} x\right\|=\inf _{y \in C}\|x-y\| .
$$

It is well-known [14] that $\mathrm{P}_{\mathrm{C}}$ is a nonexpansive mapping and is characterized by the inequality

$$
P_{C} x \in C,\left\langle x-P_{C} x, y-P_{C} x\right\rangle \leqslant 0, \quad \forall y \in C .
$$

Definition 2.1. Let $H$ be a real Hilbert space. A mapping $T: H \rightarrow H$ is said to be

(i) Lipschitzian, if there exists a constant $\rho>0$ such that

$$
\|T x-T y\| \leqslant \rho\|x-y\|, \quad \forall x, y \in H,
$$

especially, if $\rho \in(0,1), T$ is said to be a contraction with constant $\rho$;

(ii) nonexpansive, if $\|T x-T y\| \leqslant\|x-y\|$, for all $x, y \in H$;

(iii) quasi-nonexpansive, if $F(T) \neq \emptyset$ and $\|T x-q\| \leqslant\|x-q\|$, for all $x \in H, q \in F(T)$;

(iv) firmly nonexpansive, if

$$
\|T x-T y\|^{2} \leqslant\|x-y\|^{2}-\|(I-T) x-(I-T) y\|^{2}, \quad \forall x, y \in H,
$$

or equivalently,

$$
\|T x-T y\|^{2} \leqslant\langle x-y, T x-T y\rangle, \forall x, y \in H ;
$$

(v) $\mu$-demicontractive, if $F(T) \neq \emptyset$ and there exists a constant $\mu \in(-\infty, 1)$ such that

$$
\|T x-q\|^{2} \leqslant\|x-q\|^{2}+\mu\|x-T x\|^{2}, \quad \forall x \in H, q \in F(T) .
$$

Remark 2.2. Notice that every 0-demicontractive mapping is exactly quasi-nonexpansive. In particular, we say that it is quasi-strictly pseudo-contractive [9] if $0 \leqslant \mu<1$. Moreover, if $\mu \leqslant 0$, every $\mu$-demicontractive mapping becomes quasi-nonexpansive. Therefore, it is sufficient to only take $\mu \in(0,1)$ in (v) of Definition 2.1 in Hilbert spaces.

It is worth noting that the class of demicontractive mappings is more general than the class of quasinonexpansive mappings and the class of firmly quasi-nonexpansive mappings.

Definition 2.3. Let $\mathrm{C}$ be a nonempty closed convex subset of a real Hilbert space $\mathrm{H}$. A mapping $\mathrm{F}: \mathrm{C} \rightarrow \mathrm{H}$ is said to be

(i) monotone, if $\langle F x-F y, x-y\rangle \geqslant 0$, for all $x, y \in C$;

(ii) strictly monotone, if $\langle F x-F y, x-y\rangle>0$, for all $x, y \in C, x \neq y$;

(iii) $\eta$-strongly monotone, if there exists a constant $\eta>0$ such that

$$
\langle F x-F y, x-y\rangle \geqslant \eta\|x-y\|^{2}, \quad \forall x, y \in C .
$$

Definition 2.4. Let $\mathrm{H}$ be a real Hilbert space. An operator $\mathrm{T}: \mathrm{H} \rightarrow \mathrm{H}$ is called demiclosed at origin, if for any sequence $\left\{x_{k}\right\}$ which converges weakly to $x$, and if the sequence $\left\{T x_{k}\right\}$ converges strongly to 0 , then $\mathrm{T} x=0$. 
As a special case of the demicloseness principle on uniformly convex Banach spaces given by [6], we know that if $\mathrm{C}$ is a nonempty closed convex subset of a Hilbert space $\mathrm{H}$, and $\mathrm{T}: \mathrm{C} \rightarrow \mathrm{H}$ is a nonexpansive mapping, then the mapping $\mathrm{I}-\mathrm{T}$ is demiclosed on $\mathrm{C}$. Now the following question is naturally raised: If $\mathrm{T}: \mathrm{C} \rightarrow \mathrm{H}$ is quasi-nonexpansive, is $\mathrm{I}-\mathrm{T}$ still demiclosed on $\mathrm{C}$ ? The answer is negative even at 0 as follows.

Example 2.5. The mapping $T:[0,1] \rightarrow[0,1]$ is defined by

$$
T x=\left\{\begin{array}{l}
\frac{x}{5}, \quad x \in\left[0, \frac{1}{2}\right] \\
x \sin \pi x, \quad x \in\left(\frac{1}{2}, 1\right] .
\end{array}\right.
$$

Then $\mathrm{T}$ is a quasi-nonexpansive mapping, but $\mathrm{I}-\mathrm{T}$ is not demiclosed at 0 .

In fact, $F(T)=\{0\}$. For any $x \in\left[0, \frac{1}{2}\right]$, we have

$$
|\mathrm{T} x-0|=\left|\frac{x}{5}-0\right| \leqslant|x-0|
$$

and for any $x \in\left(\frac{1}{2}, 1\right]$, we have

$$
|\mathrm{T} x-0|=|x \sin \pi x-0| \leqslant|x-0| .
$$

Thus $T$ is quasi-nonexpansive. Taking $\left\{x_{n}\right\} \subset\left(\frac{1}{2}, 1\right]$ and $x_{n} \rightarrow \frac{1}{2}(n \rightarrow \infty)$, we have

$$
\left|(\mathrm{I}-\mathrm{T}) \mathrm{x}_{\mathrm{n}}\right|=\left|\mathrm{x}_{\mathrm{n}}\left[1-\sin \pi \mathrm{x}_{\mathrm{n}}\right]\right| \rightarrow 0(\mathrm{n} \rightarrow \infty) .
$$

But $\mathrm{T} \frac{1}{2}=\frac{1}{10} \neq \frac{1}{2}$, i.e., $(\mathrm{I}-\mathrm{T}) \frac{1}{2} \neq 0$, so $\mathrm{I}-\mathrm{T}$ is not demiclosed at 0 .

Lemma 2.6 ([10]). Let $\mathrm{T}$ be a $\mu$-demicontractive self-mapping on $\mathrm{H}$ with $\mathrm{F}(\mathrm{T}) \neq \emptyset$ and set $\mathrm{T}_{\alpha}=(1-\alpha) \mathrm{I}+\alpha \mathrm{T}$ for $\alpha \in[0,1]$. Then, $\mathrm{T}_{\alpha}$ is quasi-nonexpansive provided that $\alpha \in[0,1-\mu]$ and

$$
\left\|T_{\alpha} x-q\right\|^{2} \leqslant\|x-q\|^{2}-\alpha(1-\mu-\alpha)\|x-T x\|^{2}, \quad x \in H, \quad q \in F(T) .
$$

Lemma 2.7 ([9, Proposition 2.1]). Assume $\mathrm{C}$ is a closed convex subset of a Hilbert space $\mathrm{H}$. Let $\mathrm{T}: \mathrm{C} \rightarrow \mathrm{C}$ be a self-mapping of $\mathrm{C}$. If $\mathrm{T}$ is a $\mu$-demicontractive mapping (which is also called $\mu$-quasi-strict pseudo-contraction in [9]), then the fixed point set $\mathrm{F}(\mathrm{T})$ is closed and convex.

Lemma 2.8 ([7]). Assume $\left\{\mathrm{s}_{\mathrm{k}}\right\}$ is a sequence of nonnegative real numbers such that

$$
\left\{\begin{array}{l}
s_{k+1} \leqslant\left(1-\lambda_{k}\right) s_{k}+\lambda_{k} \delta_{k} \\
s_{k+1} \leqslant s_{k}-\eta_{k}+\mu_{k}
\end{array}\right.
$$

where $\left\{\lambda_{k}\right\}$ is a sequence in $(0,1),\left\{\eta_{k}\right\}$ is a sequence of nonnegative real numbers and $\left\{\delta_{k}\right\}$ and $\left\{\mu_{k}\right\}$ are two sequences in $\mathbb{R}$ such that

(i) $\sum_{k=1}^{\infty} \lambda_{k}=\infty$;

(ii) $\lim _{k \rightarrow \infty} \mu_{k}=0$;

(iii) $\lim _{l \rightarrow \infty} \eta_{k_{l}}=0$ implies $\lim \sup _{l \rightarrow \infty} \delta_{k_{l}} \leqslant 0$ for any subsequence $\left\{k_{l}\right\} \subset\{k\}$.

Then $\lim _{k \rightarrow \infty} s_{k}=0$.

Lemma 2.9 ([8]). Let $\mathrm{X}$ and $\mathrm{Y}$ be Banach spaces, $\mathrm{A}$ be a continuous linear operator from $\mathrm{X}$ to $\mathrm{Y}$. Then $\mathrm{A}$ is weakly continuous.

Lemma 2.10 ([16, Proposition 2.7]). Let $\mathrm{H}$ be a real Hilbert space. Suppose that $\mathrm{F}: \mathrm{H} \rightarrow \mathrm{H}$ is $\mathrm{k}$-Lipschitzian and $\eta$-strongly monotone over a closed convex set $\mathrm{C} \subset \mathrm{H}$. Then, the following $\operatorname{VIP}(\mathrm{F}, \mathrm{C})$

$$
\left\langle v-\mathrm{u}^{*}, \mathrm{~F}\left(\mathrm{u}^{*}\right)\right\rangle \geqslant 0, \quad \forall v \in \mathrm{C},
$$

has its unique solution $\mathrm{u}^{*} \in \mathrm{C}$. 


\section{Main results}

In this section, we introduce a new parallel algorithm combining viscosity approximation methods for the MSECFP (1.4) of demicontractive mappings and prove strong convergence of the algorithm.

Algorithm 3.1. Let $f_{1}: H_{1} \rightarrow H_{1}$ and $f_{2}: H_{2} \rightarrow H_{2}$ be two contractions with constants $\rho_{1}, \rho_{2} \in[0,1)$ and $\left\{t_{k}\right\} \subset[0,1]$. Let $x_{0} \in H_{1}, y_{0} \in H_{2}$ be arbitrary and $p, q \geqslant 1$ be integers. Let $\left\{\alpha_{k}^{i}\right\} \subset[0,1](0 \leqslant i \leqslant p)$ and $\left\{\beta_{k}^{j}\right\} \subset[0,1](0 \leqslant j \leqslant q)$ such that $\sum_{i=0}^{p} \alpha_{k}^{i}=1$ and $\sum_{j=0}^{q} \beta_{k}^{j}=1$. Assume that the $k$-th iterate $x_{k} \in H_{1}, y_{k} \in H_{2}$ has been constructed, then we calculate $(k+1)$-th iterate $\left(x_{k+1}, y_{k+1}\right)$ via the formula

$$
\left\{\begin{array}{l}
u_{k}=x_{k}-\gamma_{k} A^{*}\left(A x_{k}-B y_{k}\right) \\
x_{k+1}=t_{k} f_{1}\left(x_{k}\right)+\left(1-t_{k}\right)\left(\alpha_{k}^{0} u_{k}+\sum_{i=1}^{p} \alpha_{k}^{i} u_{i}\left(u_{k}\right)\right) \\
v_{k}=y_{k}+\gamma_{k} B^{*}\left(A x_{k}-B y_{k}\right) \\
y_{k+1}=t_{k} f_{2}\left(y_{k}\right)+\left(1-t_{k}\right)\left(\beta_{k}^{0} v_{k}+\sum_{j=1}^{q} \beta_{k}^{j} T_{j}\left(v_{k}\right)\right)
\end{array}\right.
$$

Put $\mathrm{H}^{*}=\mathrm{H}_{1} \times \mathrm{H}_{2}$. Define the inner product of $\mathrm{H}^{*}$ as follows:

$$
\left\langle\left(x_{1}, y_{1}\right),\left(x_{2}, y_{2}\right)\right\rangle=\left\langle x_{1}, x_{2}\right\rangle+\left\langle y_{1}, y_{2}\right\rangle, \quad \forall\left(x_{1}, y_{1}\right),\left(x_{2}, y_{2}\right) \in H^{*}
$$

It is easy to see that $\mathrm{H}^{*}$ is also a real Hilbert space and

$$
\|(x, y)\|=\left(\|x\|^{2}+\|y\|^{2}\right)^{\frac{1}{2}}, \quad \forall(x, y) \in H^{*} .
$$

Lemma 3.2. Given two bounded linear operators $A: \mathrm{H}_{1} \rightarrow \mathrm{H}_{3}, \mathrm{~B}: \mathrm{H}_{2} \rightarrow \mathrm{H}_{3}$, let $\mathrm{U}_{i}: \mathrm{H}_{1} \rightarrow \mathrm{H}_{1}(1 \leqslant i \leqslant p)$ and $\mathrm{T}_{\mathrm{j}}: \mathrm{H}_{2} \rightarrow \mathrm{H}_{2}(1 \leqslant j \leqslant q)$ be $\tau_{\mathfrak{i}}$-demicontractive and $\theta_{j}$-demicontractive, respectively. Assume that the solution set $\Gamma$ of (1.4) is nonempty. Then $\Gamma$ is a nonempty closed convex set.

Proof. By Lemma 2.7 we have $F\left(T_{i}\right)(1 \leqslant i \leqslant p)$ and $F\left(U_{j}\right)(1 \leqslant j \leqslant q)$ are both closed convex subsets, and since $A$ and $B$ are both linear, it is easy to see that $\Gamma$ is a closed convex subset in $\mathrm{H}^{*}$.

Theorem 3.3. Let $\mathrm{H}_{1}, \mathrm{H}_{2}, \mathrm{H}_{3}$ be real Hilbert spaces. Given two bounded linear operators $A: \mathrm{H}_{1} \rightarrow \mathrm{H}_{3}, \mathrm{~B}: \mathrm{H}_{2} \rightarrow$ $\mathrm{H}_{3}$, let $\mathrm{U}_{i}: \mathrm{H}_{1} \rightarrow \mathrm{H}_{1}(1 \leqslant i \leqslant p)$ and $\mathrm{T}_{j}: \mathrm{H}_{2} \rightarrow \mathrm{H}_{2}(1 \leqslant j \leqslant q)$ be $\tau_{i}$-demicontractive and $\theta_{j}$-demicontractive, respectively. Suppose that $\mathrm{I}-\mathrm{U}_{i}(1 \leqslant i \leqslant p), I-\mathrm{T}_{j}(1 \leqslant j \leqslant q)$ are demiclosed at origin and the solution set $\Gamma$ of the MSECFP (1.4) is nonempty. Assume that the following conditions are satisfied:

(i) $\rho_{1}, \rho_{2} \in\left[0, \frac{1}{\sqrt{2}}\right)$;

(ii) $\lim _{k \rightarrow \infty} t_{k}=0$ and $\sum_{k=0}^{\infty} t_{k}=\infty$;

(iii) $\liminf _{k \rightarrow \infty} \alpha_{k}^{0}>\tau, \liminf _{k \rightarrow \infty} \beta_{k}^{0}>\mu$;

(iv) $\liminf _{k \rightarrow \infty} \alpha_{k}^{i}>0(1 \leqslant i \leqslant p), \liminf _{k \rightarrow \infty} \beta_{k}^{j}>0(1 \leqslant j \leqslant q)$;

(v) $\gamma_{\mathrm{k}} \in\left(\varepsilon, \frac{2}{\lambda_{\mathrm{A}}+\lambda_{\mathrm{B}}}-\varepsilon\right)$,

where $\tau=\max _{1 \leqslant i \leqslant p} \tau_{i}, \mu=\max _{1 \leqslant j \leqslant q} \theta_{j}, \lambda_{A}, \lambda_{B}$ stand for the spectral radiuses of $A^{*} A$ and $B * B$, respectively and $\varepsilon>0$ is small enough. Then the sequence $\left\{\left(x_{k}, y_{k}\right)\right\}$ generated by Algorithm 3.1 converges strongly to $\left(x^{*}, y^{*}\right) \in \Gamma$ which is the unique solution of the following variational inequality problem (VIP)

$$
\left\langle\left(\left(I-f_{1}\right) x^{*},\left(I-f_{2}\right) y^{*}\right),(x, y)-\left(x^{*}, y^{*}\right)\right\rangle \geqslant 0, \quad \forall(x, y) \in \Gamma .
$$

Proof. We divide the proof into several steps.

Step 1. The VIP (3.2) has a unique solution $\left(x^{*}, y^{*}\right) \in \Gamma$. 
By Lemma 3.2, we know that $\Gamma$ is a nonempty closed convex subset in $\mathrm{H}^{*}$. Let $\mathrm{F}: \Gamma \subset \mathrm{H}^{*} \rightarrow \mathrm{H}^{*}$ be defined by

$$
F(x, y)=\left(\left(I-f_{1}\right) x,\left(I-f_{2}\right) y\right), \quad \forall(x, y) \in \Gamma .
$$

Putting $\rho=\max \left\{\rho_{1}, \rho_{2}\right\}$, then from the condition (i) we have $\rho \in\left[0, \frac{1}{\sqrt{2}}\right)$. For any $\left(x_{1}, y_{1}\right),\left(x_{2}, y_{2}\right) \in \Gamma$, since $f_{1}$ and $f_{2}$ are two contractions, we have

$$
\begin{aligned}
\left\langle F\left(x_{1}, y_{1}\right)-\right. & \left.F\left(x_{2}, y_{2}\right),\left(x_{1}, y_{1}\right)-\left(x_{2}, y_{2}\right)\right\rangle \\
= & \left\langle\left(\left(I-f_{1}\right) x_{1}-\left(I-f_{1}\right) x_{2},\left(I-f_{2}\right) y_{1}-\left(I-f_{2}\right) y_{2}\right),\left(x_{1}-x_{2}, y_{1}-y_{2}\right)\right\rangle \\
= & \left\langle\left(I-f_{1}\right) x_{1}-\left(I-f_{1}\right) x_{2}, x_{1}-x_{2}\right\rangle+\left\langle\left(I-f_{2}\right) y_{1}-\left(I-f_{2}\right) y_{2}, y_{1}-y_{2}\right\rangle \\
\geqslant & \left\|x_{1}-x_{2}\right\|^{2}-\left\|f_{1}\left(x_{1}\right)-f_{1}\left(x_{2}\right)\right\|\left\|x_{1}-x_{2}\right\| \\
& +\left\|y_{1}-y_{2}\right\|^{2}-\left\|f_{2}\left(y_{1}\right)-f_{2}\left(y_{2}\right)\right\|\left\|y_{1}-y_{2}\right\| \\
\geqslant & (1-\rho)\left(\left\|x_{1}-x_{2}\right\|^{2}+\left\|y_{1}-y_{2}\right\|^{2}\right) \\
= & (1-\rho)\left\|\left(x_{1}, y_{1}\right)-\left(x_{2}, y_{2}\right)\right\|^{2},
\end{aligned}
$$

which implies that $F$ is $(1-\rho)$-strongly monotone, and

$$
\begin{aligned}
\left\|F\left(x_{1}, y_{1}\right)-F\left(x_{2}, y_{2}\right)\right\|^{2} & =\left\|\left(\left(I-f_{1}\right) x_{1}-\left(I-f_{1}\right) x_{2},\left(I-f_{2}\right) y_{1}-\left(I-f_{2}\right) y_{2}\right)\right\|^{2} \\
& =\left\|\left(I-f_{1}\right) x_{1}-\left(I-f_{1}\right) x_{2}\right\|^{2}+\left\|\left(I-f_{2}\right) y_{1}-\left(I-f_{2}\right) y_{2}\right\|^{2} \\
& \leqslant 2\left(1+\rho_{1}^{2}\right)\left\|x_{1}-x_{2}\right\|^{2}+2\left(1+\rho_{2}^{2}\right)\left\|y_{1}-y_{2}\right\|^{2} \\
& \leqslant 2\left(1+\rho^{2}\right)\left\|\left(x_{1}, y_{1}\right)-\left(x_{2}, y_{2}\right)\right\|^{2},
\end{aligned}
$$

which implies that $F$ is $2\left(1+\rho^{2}\right)$-Lipschitzian. Therefore, it follows from Lemma 2.10 that the VIP (3.2) has a unique solution $\left(x^{*}, y^{*}\right) \in \Gamma$.

Step 2. The sequences $\left\{x_{n}\right\}$ and $\left\{y_{n}\right\}$ are bounded.

Since $\left(x^{*}, y^{*}\right) \in \Gamma$, then $x^{*} \in \cap_{i=1}^{p} F\left(U_{i}\right), y^{*} \in \cap_{j=1}^{q} F\left(T_{j}\right)$ such that $A x^{*}=B y^{*}$. By (3.1) and the definitions of $\lambda_{A}$ and $\lambda_{B}$, we have

$$
\begin{aligned}
\left\|u_{k}-x^{*}\right\|^{2}= & \left\|x_{k}-\gamma_{k} A^{*}\left(A x_{k}-B y_{k}\right)-x^{*}\right\|^{2} \\
= & \left\|x_{k}-x^{*}\right\|^{2}-2 \gamma_{k}\left\langle x_{k}-x^{*}, A^{*}\left(A x_{k}-B y_{k}\right)\right\rangle+\gamma_{k}^{2}\left\|A^{*}\left(A x_{k}-B y_{k}\right)\right\|^{2} \\
= & \left\|x_{k}-x^{*}\right\|^{2}-2 \gamma_{k}\left\langle A x_{k}-A x^{*}, A x_{k}-B y_{k}\right\rangle \\
& +\gamma_{k}^{2}\left\langle A^{*}\left(A x_{k}-B y_{k}\right), A^{*}\left(A x_{k}-B y_{k}\right)\right\rangle \\
= & \left\|x_{k}-x^{*}\right\|^{2}-2 \gamma_{k}\left\langle A x_{k}-A x^{*}, A x_{k}-B y_{k}\right\rangle \\
& +\gamma_{k}^{2}\left\langle A x_{k}-B y_{k}, A A^{*}\left(A x_{k}-B y_{k}\right)\right\rangle \\
\leqslant & \left\|x_{k}-x^{*}\right\|^{2}-2 \gamma_{k}\left\langle A x_{k}-A x^{*}, A x_{k}-B y_{k}\right\rangle+\gamma_{k}^{2} \lambda_{A}\left\|A x_{k}-B y_{k}\right\|^{2},
\end{aligned}
$$

and

$$
\begin{aligned}
\left\|v_{k}-y^{*}\right\|^{2}= & \left\|y_{k}+\gamma_{k} B^{*}\left(A x_{k}-B y_{k}\right)-y^{*}\right\|^{2} \\
= & \left\|y_{k}-y^{*}\right\|^{2}+2 \gamma_{k}\left\langle B y_{k}-B y^{*}, A x_{k}-B y_{k}\right\rangle+\gamma_{k}^{2}\left\|B^{*}\left(A x_{k}-B y_{k}\right)\right\|^{2} \\
= & \left\|y_{k}-y^{*}\right\|^{2}+2 \gamma_{k}\left\langle B y_{k}-B y^{*}, A x_{k}-B y_{k}\right\rangle \\
& +\gamma_{k}^{2}\left\langle A x_{k}-B y_{k}, B B^{*}\left(A x_{k}-B y_{k}\right)\right\rangle \\
\leqslant & \left\|y_{k}-y^{*}\right\|^{2}+2 \gamma_{k}\left\langle B y_{k}-B y^{*}, A x_{k}-B y_{k}\right\rangle+\gamma_{k}^{2} \lambda_{B}\left\|A x_{k}-B y_{k}\right\|^{2} .
\end{aligned}
$$


By adding the above inequalities and $A x^{*}=B y^{*}$, we have

$$
\left\|u_{k}-x^{*}\right\|^{2}+\left\|v_{k}-y^{*}\right\|^{2} \leqslant\left\|x_{k}-x^{*}\right\|^{2}+\left\|y_{k}-y^{*}\right\|^{2}-\gamma_{k}\left[2-\left(\lambda_{A}+\lambda_{B}\right) \gamma_{k}\right]\left\|A x_{k}-B y_{k}\right\|^{2} .
$$

Taking $\omega_{k}^{i}=\frac{\alpha_{k}^{i}}{1-\alpha_{k}^{0}}(1 \leqslant i \leqslant p)$ and $\widetilde{\omega}_{k}^{j}=\frac{\beta_{k}^{j}}{1-\beta_{k}^{0}}(1 \leqslant j \leqslant q)$, we have $\sum_{i=1}^{p} \omega_{k}^{i}=1$ and $\sum_{j=1}^{q} \widetilde{\omega}_{k}^{j}=1$ for every $k \geqslant 0$. Put $\widetilde{u}_{k}=\alpha_{k}^{0} u_{k}+\sum_{i=1}^{p} \alpha_{k}^{i} u_{i}\left(u_{k}\right)$ and $\widetilde{v}_{k}=\beta_{k}^{0} v_{k}+\sum_{j=1}^{q} \beta_{k}^{j} T_{j}\left(v_{k}\right)$. Then

$$
\begin{aligned}
\widetilde{u}_{k} & =\alpha_{k}^{0} u_{k}+\left(1-\alpha_{k}^{0}\right) \sum_{i=1}^{p} \omega_{k}^{i} u_{i}\left(u_{k}\right) \\
& =\sum_{i=1}^{p} \omega_{k}^{i}\left(\alpha_{k}^{0} u_{k}+\left(1-\alpha_{k}^{0}\right) u_{i}\left(u_{k}\right)\right) .
\end{aligned}
$$

Using Lemma 2.6 for any $i \in\{1,2, \cdots, p\}$, we have

$$
\begin{aligned}
\left\|\alpha_{k}^{0} u_{k}+\left(1-\alpha_{k}^{0}\right) u_{i}\left(u_{k}\right)-x^{*}\right\|^{2} & \leqslant\left\|u_{k}-x^{*}\right\|^{2}-\left(1-\alpha_{k}^{0}\right)\left(\alpha_{k}^{0}-\tau_{i}\right)\left\|u_{i}\left(u_{k}\right)-u_{k}\right\|^{2} \\
& \leqslant\left\|u_{k}-x^{*}\right\|^{2}-\left(1-\alpha_{k}^{0}\right)\left(\alpha_{k}^{0}-\tau\right)\left\|u_{i}\left(u_{k}\right)-u_{k}\right\|^{2}
\end{aligned}
$$

for all sufficiently large $k$. Thus by (3.4), (3.5), the convexity of $\|\cdot\|^{2}$ and the condition (iii) we obtain

$$
\begin{aligned}
\left\|\widetilde{\mathfrak{u}}_{k}-\chi^{*}\right\|^{2} & \leqslant \sum_{i=1}^{p} \omega_{k}^{i}\left\|\alpha_{k}^{0} u_{k}+\left(1-\alpha_{k}^{0}\right) u_{i}\left(u_{k}\right)-x^{*}\right\|^{2} \\
& \leqslant \sum_{i=1}^{p} \omega_{k}^{i}\left(\left\|u_{k}-x^{*}\right\|^{2}-\left(1-\alpha_{k}^{0}\right)\left(\alpha_{k}^{0}-\tau\right)\left\|u_{i}\left(u_{k}\right)-u_{k}\right\|^{2}\right) \\
& =\left\|u_{k}-\chi^{*}\right\|^{2}-\left(1-\alpha_{k}^{0}\right)\left(\alpha_{k}^{0}-\tau\right) \sum_{i=1}^{p} \omega_{k}^{i}\left\|u_{i}\left(u_{k}\right)-u_{k}\right\|^{2} \\
& =\left\|u_{k}-\chi^{*}\right\|^{2}-\left(\alpha_{k}^{0}-\tau\right) \sum_{i=1}^{p} \alpha_{k}^{i}\left\|u_{i}\left(u_{k}\right)-u_{k}\right\|^{2} \\
& \leqslant\left\|u_{k}-\chi^{*}\right\|^{2}
\end{aligned}
$$

for all sufficiently large $k$. Similarly, we obtain

$$
\begin{aligned}
\left\|\widetilde{v}_{k}-y^{*}\right\|^{2} & \leqslant\left\|v_{k}-y^{*}\right\|^{2}-\left(\beta_{k}^{0}-\mu\right) \sum_{j=1}^{q} \beta_{k}^{j}\left\|T_{j}\left(v_{k}\right)-v_{k}\right\|^{2} \\
& \leqslant\left\|v_{k}-y^{*}\right\|^{2}
\end{aligned}
$$

for all sufficiently large $k$. It follows from (3.1) and (3.6) that

$$
\begin{aligned}
\left\|x_{k+1}-x^{*}\right\|^{2} \leqslant & t_{k}\left\|f_{1}\left(x_{k}\right)-x^{*}\right\|^{2}+\left(1-t_{k}\right)\left\|\tilde{u}_{k}-x^{*}\right\|^{2} \\
\leqslant & t_{k}\left[\rho_{1}\left\|x_{k}-x^{*}\right\|+\left\|f_{1}\left(x^{*}\right)-x^{*}\right\|\right]^{2}+\left(1-t_{k}\right)\left(\left\|u_{k}-x^{*}\right\|^{2}\right. \\
& \left.-\left(\alpha_{k}^{0}-\tau\right) \sum_{i=1}^{p} \alpha_{k}^{i}\left\|u_{i}\left(u_{k}\right)-u_{k}\right\|^{2}\right) \\
\leqslant & 2 t_{k} \rho^{2}\left\|x_{k}-x^{*}\right\|^{2}+2 t_{k}\left\|f_{1}\left(x^{*}\right)-x^{*}\right\|^{2}+\left(1-t_{k}\right)\left\|u_{k}-x^{*}\right\|^{2} \\
& -\left(1-t_{k}\right)\left(\alpha_{k}^{0}-\tau\right) \sum_{i=1}^{p} \alpha_{k}^{i}\left\|u_{i}\left(u_{k}\right)-u_{k}\right\|^{2} .
\end{aligned}
$$

Similarly, we obtain

$$
\begin{aligned}
\left\|y_{k+1}-y^{*}\right\|^{2} \leqslant & 2 t_{k} \rho^{2}\left\|y_{k}-y^{*}\right\|^{2}+2 t_{k}\left\|f_{2}\left(y^{*}\right)-y^{*}\right\|^{2}+\left(1-t_{k}\right)\left\|v_{k}-y^{*}\right\|^{2} \\
& -\left(1-t_{k}\right)\left(\beta_{k}^{0}-\mu\right) \sum_{j=1}^{q} \beta_{k}^{j}\left\|T_{j}\left(v_{k}\right)-v_{k}\right\|^{2} .
\end{aligned}
$$


It follows from (3.3), (3.10) and (3.11) that

$$
\begin{aligned}
\left\|x_{k+1}-x^{*}\right\|^{2}+\left\|y_{k+1}-y^{*}\right\|^{2} \leqslant & 2 t_{k} \rho^{2}\left(\left\|x_{k}-x^{*}\right\|^{2}+\left\|y_{k}-y^{*}\right\|^{2}\right)+2 t_{k}\left(\left\|f_{1}\left(x^{*}\right)-x^{*}\right\|^{2}\right. \\
& \left.+\left\|f_{2}\left(y^{*}\right)-y^{*}\right\|^{2}\right)+\left(1-t_{k}\right)\left(\left\|u_{k}-x^{*}\right\|^{2}+\left\|v_{k}-y^{*}\right\|^{2}\right) \\
& -\left(1-t_{k}\right)\left(\alpha_{k}^{0}-\tau\right) \sum_{i=1}^{p} \alpha_{k}^{i}\left\|u_{i}\left(u_{k}\right)-u_{k}\right\|^{2} \\
& -\left(1-t_{k}\right)\left(\beta_{k}^{0}-\mu\right) \sum_{j=1}^{q} \beta_{k}^{j}\left\|T_{j}\left(v_{k}\right)-v_{k}\right\|^{2} \\
\leqslant & {\left[1-t_{k}\left(1-2 \rho^{2}\right)\right]\left(\left\|x_{k}-x^{*}\right\|^{2}+\left\|y_{k}-y^{*}\right\|^{2}\right) } \\
& +2 t_{k}\left(\left\|f_{1}\left(x^{*}\right)-x^{*}\right\|^{2}+\left\|f_{2}\left(y^{*}\right)-y^{*}\right\|^{2}\right) \\
& -\left(1-t_{k}\right) \gamma_{k}\left[2-\left(\lambda_{A}+\lambda_{B}\right) \gamma_{k}\right]\left\|A x_{k}-B y_{k}\right\|^{2} \\
& -\left(1-t_{k}\right)\left(\alpha_{k}^{0}-\tau\right) \sum_{i=1}^{p} \alpha_{k}^{i}\left\|u_{i}\left(u_{k}\right)-u_{k}\right\|^{2} \\
& -\left(1-t_{k}\right)\left(\beta_{k}^{0}-\mu\right) \sum_{j=1}^{q} \beta_{k}^{j}\left\|T_{j}\left(v_{k}\right)-v_{k}\right\|^{2} .
\end{aligned}
$$

Then setting $s_{k}=\left\|x_{k}-x^{*}\right\|^{2}+\left\|y_{k}-y^{*}\right\|^{2}$, we get

$$
\begin{aligned}
s_{k+1} \leqslant & {\left[1-t_{k}\left(1-2 \rho^{2}\right)\right] s_{k}+2 t_{k}\left(\left\|f_{1}\left(x^{*}\right)-x^{*}\right\|^{2}+\left\|f_{2}\left(y^{*}\right)-y^{*}\right\|^{2}\right) } \\
& -\left(1-t_{k}\right) \gamma_{k}\left[2-\left(\lambda_{A}+\lambda_{B}\right) \gamma_{k}\right]\left\|A x_{k}-B y_{k}\right\|^{2} \\
& -\left(1-t_{k}\right)\left(\alpha_{k}^{0}-\tau\right) \sum_{i=1}^{p} \alpha_{k}^{i}\left\|u_{i}\left(u_{k}\right)-u_{k}\right\|^{2} \\
& -\left(1-t_{k}\right)\left(\beta_{k}^{0}-\mu\right) \sum_{j=1}^{q} \beta_{k}^{j}\left\|T_{j}\left(v_{k}\right)-v_{k}\right\|^{2} \\
\leqslant & {\left[1-t_{k}\left(1-2 \rho^{2}\right)\right] s_{k}+t_{k}\left(1-2 \rho^{2}\right) \frac{2\left(\left\|f_{1}\left(x^{*}\right)-x^{*}\right\|^{2}+\left\|f_{2}\left(y^{*}\right)-y^{*}\right\|^{2}\right)}{1-2 \rho^{2}} . }
\end{aligned}
$$

It follows from induction that

$$
s_{k+1} \leqslant \max \left\{s_{0}, \frac{2\left(\left\|f_{1}\left(x^{*}\right)-x^{*}\right\|^{2}+\left\|f_{2}\left(y^{*}\right)-y^{*}\right\|^{2}\right)}{1-2 \rho^{2}}\right\}, \quad \forall k \geqslant 0,
$$

i.e., $\left\{s_{k}\right\}$ is bounded. So $\left\{x_{k}\right\}$ and $\left\{y_{k}\right\}$ are also bounded.

Step 3. The sequence $\left\{\left(x_{k}, y_{k}\right)\right\}$ converges strongly to $\left(x^{*}, y^{*}\right)$.

It follows from (3.1) and (3.7) that

$$
\begin{aligned}
\left\|x_{k+1}-x^{*}\right\|^{2}= & t_{k}^{2}\left\|f_{1}\left(x_{k}\right)-x^{*}\right\|^{2}+2 t_{k}\left(1-t_{k}\right)\left\langle f_{1}\left(x_{k}\right)-x^{*}, \widetilde{u}_{k}-x^{*}\right\rangle \\
& +\left(1-t_{k}\right)^{2}\left\|\widetilde{u}_{k}-x^{*}\right\|^{2} \\
\leqslant & t_{k}^{2}\left\|f_{1}\left(x_{k}\right)-x^{*}\right\|^{2}+t_{k}\left(1-t_{k}\right)\left(\left\|f_{1}\left(x_{k}\right)-f_{1}\left(x^{*}\right)\right\|^{2}+\left\|\widetilde{u}_{k}-x^{*}\right\|^{2}\right) \\
& +\left(1-t_{k}\right)^{2}\left\|\widetilde{u}_{k}-x^{*}\right\|^{2}+2 t_{k}\left(1-t_{k}\right)\left\langle f_{1}\left(x^{*}\right)-x^{*}, \widetilde{u}_{k}-x^{*}\right\rangle \\
\leqslant & t_{k}^{2}\left\|f_{1}\left(x_{k}\right)-x^{*}\right\|^{2}+t_{k}\left(1-t_{k}\right)\left(\rho_{1}^{2}\left\|x_{k}-x^{*}\right\|^{2}+\left\|u_{k}-x^{*}\right\|^{2}\right) \\
& +\left(1-t_{k}\right)^{2}\left\|u_{k}-x^{*}\right\|^{2}+2 t_{k}\left(1-t_{k}\right)\left\langle f_{1}\left(x^{*}\right)-x^{*}, \widetilde{u}_{k}-x^{*}\right\rangle \\
\leqslant & t_{k}\left(1-t_{k}\right) \rho^{2}\left\|x_{k}-x^{*}\right\|^{2}+\left(1-t_{k}\right)\left\|u_{k}-x^{*}\right\|^{2} \\
& +t_{k}^{2}\left\|f_{1}\left(x_{k}\right)-x^{*}\right\|^{2}+2 t_{k}\left(1-t_{k}\right)\left\langle f_{1}\left(x^{*}\right)-x^{*}, \widetilde{u}_{k}-x^{*}\right\rangle .
\end{aligned}
$$


Similarly we have

$$
\begin{aligned}
\left\|y_{k+1}-y^{*}\right\|^{2} \leqslant & t_{k}\left(1-t_{k}\right) \rho^{2}\left\|y_{k}-y^{*}\right\|^{2}+\left(1-t_{k}\right)\left\|v_{k}-y^{*}\right\|^{2}+t_{k}^{2}\left\|f_{2}\left(y_{k}\right)-y^{*}\right\|^{2} \\
& +2 t_{k}\left(1-t_{k}\right)\left\langle f_{2}\left(y^{*}\right)-y^{*}, \widetilde{v}_{k}-y^{*}\right\rangle .
\end{aligned}
$$

By (3.3), (3.13) and (3.14) we get

$$
\begin{aligned}
s_{k+1} \leqslant & t_{k}\left(1-t_{k}\right) \rho^{2}\left(\left\|x_{k}-x^{*}\right\|^{2}+\left\|y_{k}-y^{*}\right\|^{2}\right) \\
& +\left(1-t_{k}\right)\left(\left\|u_{k}-x^{*}\right\|^{2}+\left\|v_{k}-y^{*}\right\|^{2}\right) \\
& +t_{k}^{2}\left(\left\|f_{1}\left(x_{k}\right)-x^{*}\right\|^{2}+\left\|f_{2}\left(y_{k}\right)-y^{*}\right\|^{2}\right) \\
& +2 t_{k}\left(1-t_{k}\right)\left(\left\langle f_{1}\left(x^{*}\right)-x^{*}, \widetilde{u}_{k}-x^{*}\right\rangle+\left\langle f_{2}\left(y^{*}\right)-y^{*}, \widetilde{v}_{k}-y^{*}\right\rangle\right) \\
\leqslant & {\left[1-t_{k}\left(1-\left(1-t_{k}\right) \rho^{2}\right)\right] s_{k}+t_{k}^{2}\left(\left\|f_{1}\left(x_{k}\right)-x^{*}\right\|^{2}+\left\|f_{2}\left(y_{k}\right)-y^{*}\right\|^{2}\right) } \\
& +2 t_{k}\left(1-t_{k}\right)\left(\left\langle f_{1}\left(x^{*}\right)-x^{*}, \widetilde{u}_{k}-x^{*}\right\rangle+\left\langle f_{2}\left(y^{*}\right)-y^{*}, \widetilde{v}_{k}-y^{*}\right\rangle\right) \\
= & \left(1-\lambda_{k}\right) s_{k}+\lambda_{k} \delta_{k},
\end{aligned}
$$

where $\lambda_{k}=t_{k}\left(1-\left(1-t_{k}\right) \rho^{2}\right)$,

$$
\begin{aligned}
\delta_{k}= & \frac{t_{k}\left(\left\|f_{1}\left(x_{k}\right)-x^{*}\right\|^{2}+\left\|f_{2}\left(y_{k}\right)-y^{*}\right\|^{2}\right)}{1-\left(1-t_{k}\right) \rho^{2}} \\
& +\frac{2\left(1-t_{k}\right)\left(\left\langle f_{1}\left(x^{*}\right)-x^{*}, \widetilde{u}_{k}-x^{*}\right\rangle+\left\langle f_{2}\left(y^{*}\right)-y^{*}, \widetilde{v}_{k}-y^{*}\right\rangle\right)}{1-\left(1-t_{k}\right) \rho^{2}} .
\end{aligned}
$$

From (3.1), (3.6) and (3.8) we have

$$
\begin{aligned}
\left\|x_{k+1}-x^{*}\right\|^{2} \leqslant & t_{k}\left\|f_{1}\left(x_{k}\right)-x^{*}\right\|^{2}+\left(1-t_{k}\right)\left\|\tilde{u}_{k}-x^{*}\right\|^{2} \\
\leqslant & t_{k}\left\|f_{1}\left(x_{k}\right)-x^{*}\right\|^{2}+\left(1-t_{k}\right)\left(\left\|u_{k}-x^{*}\right\|^{2}\right. \\
& \left.-\left(\alpha_{k}^{0}-\tau\right) \sum_{i=1}^{p} \alpha_{k}^{i}\left\|u_{i}\left(u_{k}\right)-u_{k}\right\|^{2}\right),
\end{aligned}
$$

and

$$
\begin{aligned}
\left\|y_{k+1}-x^{*}\right\|^{2} \leqslant & t_{k}\left\|f_{2}\left(y_{k}\right)-y^{*}\right\|^{2}+\left(1-t_{k}\right)\left\|\widetilde{v}_{k}-y^{*}\right\|^{2} \\
\leqslant & t_{k}\left\|f_{2}\left(y_{k}\right)-y^{*}\right\|^{2}+\left(1-t_{k}\right)\left(\left\|v_{k}-y^{*}\right\|^{2}\right. \\
& \left.-\left(\beta_{k}^{0}-\mu\right) \sum_{j=1}^{q} \beta_{k}^{j}\left\|T_{j}\left(v_{k}\right)-v_{k}\right\|^{2}\right),
\end{aligned}
$$

which together with (3.3) imply that

$$
\begin{aligned}
s_{k+1} \leqslant & \left\|u_{k}-x^{*}\right\|^{2}+\left\|v_{k}-y^{*}\right\|^{2}+t_{k}\left(\left\|f_{1}\left(x_{k}\right)-x^{*}\right\|^{2}+\left\|f_{2}\left(y_{k}\right)-y^{*}\right\|^{2}\right) \\
& -\left(1-t_{k}\right)\left(\left(\alpha_{k}^{0}-\tau\right) \sum_{i=1}^{p} \alpha_{k}^{i}\left\|u_{i}\left(u_{k}\right)-u_{k}\right\|^{2}\right. \\
& \left.+\left(\beta_{k}^{0}-\mu\right) \sum_{j=1}^{q} \beta_{k}^{j}\left\|T_{j}\left(v_{k}\right)-v_{k}\right\|^{2}\right) \\
\leqslant & s_{k}+t_{k}\left(\left\|f_{1}\left(x_{k}\right)-x^{*}\right\|^{2}+\left\|f_{2}\left(y_{k}\right)-y^{*}\right\|^{2}\right)-\gamma_{k}\left[2-\left(\lambda_{A}+\lambda_{B}\right) \gamma_{k}\right] \\
& \times\left\|A x_{k}-B y_{k}\right\|^{2}-\left(1-t_{k}\right)\left(\left(\alpha_{k}^{0}-\tau\right)\right. \\
& \left.\times \sum_{i=1}^{p} \alpha_{k}^{i}\left\|u_{i}\left(u_{k}\right)-u_{k}\right\|^{2}+\left(\beta_{k}^{0}-\mu\right) \sum_{j=1}^{q} \beta_{k}^{j}\left\|T_{j}\left(v_{k}\right)-v_{k}\right\|^{2}\right) \\
\leqslant & s_{k}-\eta_{k}+\mu_{k},
\end{aligned}
$$


where $\mu_{k}=t_{k}\left(\left\|f_{1}\left(x_{k}\right)-x^{*}\right\|^{2}+\left\|f_{2}\left(y_{k}\right)-y^{*}\right\|^{2}\right)$,

$$
\begin{aligned}
\eta_{k}= & \gamma_{k}\left[2-\left(\lambda_{A}+\lambda_{B}\right) \gamma_{k}\right]\left\|A x_{k}-B y_{k}\right\|^{2}+\left(1-t_{k}\right)\left(\left(\alpha_{k}^{0}-\tau\right)\right. \\
& \left.\times \sum_{i=1}^{p} \alpha_{k}^{i}\left\|u_{i}\left(u_{k}\right)-u_{k}\right\|^{2}+\left(\beta_{k}^{0}-\mu\right) \sum_{j=1}^{q} \beta_{k}^{j}\left\|T_{j}\left(v_{k}\right)-v_{k}\right\|^{2}\right) .
\end{aligned}
$$

It follows that $\sum_{k=0}^{\infty} \lambda_{k}$ and $\lim _{k \rightarrow \infty} \mu_{k}=0$ due to the condition (ii) and the boundedness of $\left\{x_{k}\right\}$ and $\left\{y_{k}\right\}$.

Next we show that $\lim _{l \rightarrow \infty} \eta_{k_{l}}=0$ implies that $\limsup _{l \rightarrow \infty} \delta_{k_{l}} \leqslant 0$ for any $\left\{k_{l}\right\} \subset\{k\}$. Indeed, for any $\left\{k_{l}\right\} \subset\{k\}$ and $\lim _{l \rightarrow \infty} \eta_{k_{l}}=0$, by the conditions (ii)-(v), for any $i \in\{1,2, \cdots, p\}, j \in\{1,2, \cdots, q\}$ we have

$$
\lim _{l \rightarrow \infty}\left\|A x_{k_{l}}-B y_{k_{l}}\right\|=\lim _{l \rightarrow \infty}\left\|u_{k_{l}}-u_{i}\left(u_{k_{l}}\right)\right\|=\lim _{l \rightarrow \infty}\left\|v_{k_{l}}-T_{j}\left(v_{k_{l}}\right)\right\|=0 .
$$

Then we have

$$
\begin{aligned}
& \lim _{l \rightarrow \infty}\left\|u_{k_{l}}-x_{k_{l}}\right\|=\lim _{l \rightarrow \infty} \gamma_{k_{l}}\left\|A^{*}\left(A x_{k_{l}}-B y_{k_{l}}\right)\right\|=0, \\
& \lim _{l \rightarrow \infty}\left\|v_{k_{l}}-y_{k_{l}}\right\|=\lim _{l \rightarrow \infty} \gamma_{k_{l}}\left\|B^{*}\left(A x_{k_{l}}-B y_{k_{l}}\right)\right\|=0 .
\end{aligned}
$$

For any $(\widetilde{x}, \widetilde{y}) \in \omega_{w}\left(x_{k_{l}}, y_{k_{l}}\right)$, from (3.18) and (3.19) we have $(\widetilde{x}, \widetilde{y}) \in \omega_{w}\left(u_{k_{l}}, v_{k_{l}}\right)$. Due to the demiclosedness of $I-U_{i}(1 \leqslant i \leqslant p)$ and $I-T_{j}(1 \leqslant j \leqslant q)$ at origin and (3.17) we get $\widetilde{x} \in \cap_{i=1}^{p} F\left(U_{i}\right)$ and $\widetilde{y} \in \cap_{j=1}^{q} F\left(T_{j}\right)$. It follows from Lemma 2.9 that $A \widetilde{x}-B \widetilde{y} \in \omega_{w}\left(A x_{k_{l}}-B y_{k_{l}}\right)$, which together with the weakly lower semicontinuity of the norm and (3.17) implies

$$
\|A \widetilde{x}-B \widetilde{y}\| \leqslant \liminf _{l \rightarrow \infty}\left\|A x_{k_{l}}-B y_{k_{l}}\right\|=0 .
$$

Hence $(\widetilde{x}, \widetilde{y}) \in \Gamma$, i.e., $\omega_{w}\left(x_{k_{l}}, y_{k_{l}}\right) \subset \Gamma$. It is easy to see that $\lim _{k \rightarrow \infty}\left(1-\left(1-t_{k}\right) \rho^{2}\right)=1-\rho^{2}$ and $\lim _{k \rightarrow \infty} t_{k}\left(\left\|f_{1}\left(x_{k}\right)-x^{*}\right\|^{2}+\left\|f_{2}\left(y_{k}\right)-y^{*}\right\|^{2}\right)=0$. So finally we only need to prove

$$
\limsup _{l \rightarrow \infty}\left(\left\langle f_{1}\left(x^{*}\right)-x^{*}, \widetilde{u}_{k_{l}}-x^{*}\right\rangle+\left\langle f_{2}\left(y^{*}\right)-y^{*}, \widetilde{v}_{k_{l}}-y^{*}\right\rangle\right) \leqslant 0 .
$$

From (3.17)-(3.19), for any $i \in\{1,2, \cdots, p\}, j \in\{1,2, \cdots, q\}$, we have

$$
\lim _{l \rightarrow \infty}\left\|u_{i}\left(u_{k_{l}}\right)-x_{k_{l}}\right\|=\lim _{l \rightarrow \infty}\left\|T_{j}\left(v_{k_{l}}\right)-y_{k_{l}}\right\|=0,
$$

furthermore, by (3.18) and (3.19) we obtain

$$
\begin{aligned}
& \limsup _{l \rightarrow \infty}\left(\left\langle f_{1}\left(x^{*}\right)-x^{*}, \widetilde{u}_{k_{l}}-x^{*}\right\rangle+\left\langle f_{2}\left(y^{*}\right)-y^{*}, \widetilde{v}_{k_{l}}-y^{*}\right\rangle\right) \\
& =\limsup _{l \rightarrow \infty}\left(\left\langle f_{1}\left(x^{*}\right)-x^{*}, \alpha_{k_{l}}^{0} u_{k_{l}}+\sum_{i=1}^{p} \alpha_{k_{l}}^{i} u_{i}\left(u_{k_{l}}\right)-x^{*}\right\rangle\right. \\
& \left.+\left\langle\mathrm{f}_{2}\left(\mathrm{y}^{*}\right)-\mathrm{y}^{*}, \beta_{\mathrm{k}_{\mathrm{l}}}^{0} v_{\mathrm{k}_{l}}+\sum_{j=1}^{\mathrm{q}} \beta_{\mathrm{k}_{\mathrm{l}}}^{j} \mathrm{~T}_{\mathrm{j}}\left(v_{\mathrm{k}_{\mathrm{l}}}\right)-\mathrm{y}^{*}\right\rangle\right) \\
& \leqslant \limsup _{l \rightarrow \infty}\left(\left\langle f_{1}\left(x^{*}\right)-x^{*}, \alpha_{k_{l}}^{0} x_{k_{l}}+\sum_{i=1}^{p} \alpha_{k_{l}}^{i} x_{k_{l}}-x^{*}\right\rangle\right. \\
& \left.+\left\langle f_{2}\left(y^{*}\right)-y^{*}, \beta_{k_{l}}^{0} y_{k_{l}}+\sum_{j=1}^{q} \beta_{k_{l}}^{j} y_{k_{l}}-y^{*}\right\rangle\right) \\
& +\limsup _{l \rightarrow \infty}\left\langle f_{1}\left(x^{*}\right)-x^{*}, \alpha_{k_{l}}^{0}\left(u_{k_{l}}-x_{k_{l}}\right)+\sum_{i=1}^{p} \alpha_{k_{l}}^{i}\left(u_{i}\left(u_{k_{l}}\right)-x_{k_{l}}\right)\right\rangle \\
& +\limsup _{l \rightarrow \infty}\left\langle f_{2}\left(y^{*}\right)-y^{*}, \beta_{k_{l}}^{0}\left(v_{k_{l}}-y_{k_{l}}\right)+\sum_{j=1}^{q} \beta_{k_{l}}^{j}\left(T_{j}\left(v_{k_{l}}\right)-y_{k_{l}}\right)\right\rangle \\
& \leqslant \limsup _{l \rightarrow \infty}\left(\left\langle f_{1}\left(x^{*}\right)-x^{*}, x_{k_{l}}-x^{*}\right\rangle+\left\langle f_{2}\left(y^{*}\right)-y^{*}, y_{k_{l}}-y^{*}\right\rangle\right) \text {. }
\end{aligned}
$$


By the boundedness of $\left\{\left(x_{k_{l}}, y_{k_{l}}\right)\right\}$ in $H^{*}$, there exists a point $\left(p^{*}, q^{*}\right) \in H^{*}$ and a subsequence $\left\{\left(x_{k_{1}^{\prime}}, y_{k_{l}^{\prime}}\right)\right\}$ of $\left\{\left(x_{k_{l}}, y_{k_{l}}\right)\right\}$ in $H^{*}$ such that $\left(x_{k_{1}^{\prime}}, y_{k_{l}^{\prime}}\right) \rightarrow\left(p^{*}, q^{*}\right)$ and

$$
\begin{aligned}
\limsup _{l \rightarrow \infty}\left(\left\langle f_{1}\left(x^{*}\right)-x^{*}, x_{k_{l}}-x^{*}\right\rangle\right. & \left.+\left\langle f_{2}\left(y^{*}\right)-y^{*}, y_{k_{l}}-y^{*}\right\rangle\right) \\
& =\lim _{l \rightarrow \infty}\left(\left\langle f_{1}\left(x^{*}\right)-x^{*}, x_{k_{l}^{\prime}}-x^{*}\right\rangle+\left\langle f_{2}\left(y^{*}\right)-y^{*}, y_{k_{l}^{\prime}}-y^{*}\right\rangle\right) .
\end{aligned}
$$

Then $\left(p^{*}, q^{*}\right) \in \omega_{w}\left(x_{k_{l}}, y_{k_{l}}\right)$. Similar to the proof of $(\widetilde{x}, \widetilde{y}) \in \Gamma$, we have $\left(p^{*}, q^{*}\right) \in \Gamma$. Thus by (3.2), (3.20) and (3.21) we obtain

$$
\begin{aligned}
\limsup _{l \rightarrow \infty}\left(\left\langle f_{1}\left(x^{*}\right)-x^{*}, \widetilde{u}_{k_{l}}-x^{*}\right\rangle\right. & \left.+\left\langle f_{2}\left(y^{*}\right)-y^{*}, \widetilde{v}_{k_{l}}-y^{*}\right\rangle\right) \\
& \leqslant \lim _{l \rightarrow \infty}\left(\left\langle f_{1}\left(x^{*}\right)-x^{*}, x_{k_{l}^{\prime}}-x^{*}\right\rangle+\left\langle f_{2}\left(y^{*}\right)-y^{*}, y_{k_{l}^{\prime}}-y^{*}\right\rangle\right) \\
& =\left\langle f_{1}\left(x^{*}\right)-x^{*}, p^{*}-x^{*}\right\rangle+\left\langle f_{2}\left(y^{*}\right)-y^{*}, q^{*}-y^{*}\right\rangle \\
& =-\left\langle\left(I-f_{1}\right) x^{*}-\left(I-f_{2}\right) y^{*},\left(p^{*}, q^{*}\right)-\left(x^{*}, y^{*}\right)\right\rangle \leqslant 0
\end{aligned}
$$

i.e., $\lim \sup _{l \rightarrow \infty} \delta_{k_{l}} \leqslant 0$. Therefore it follows from Lemma 2.8 that $\lim _{k \rightarrow \infty} s_{k}=0$, that is

$$
\lim _{k \rightarrow \infty}\left(\left\|x_{k}-x^{*}\right\|^{2}+\left\|y_{k}-y^{*}\right\|^{2}\right)=0,
$$

which implies that $\left\{\left(x_{k}, y_{k}\right)\right\}$ generated by Algorithm 3.1 converges strongly to $\left(x^{*}, y^{*}\right) \in \Gamma$ which is the unique solution of the VIP (3.2).

Take $\mathrm{U}_{1}=\mathrm{U}_{2}=\cdots=\mathrm{U}_{\mathrm{p}}=\mathrm{U}, \mathrm{T}_{1}=\mathrm{T}_{2}=\cdots=\mathrm{T}_{\mathrm{q}}=\mathrm{T}$. Then Algorithm 3.1 reduces to the following algorithm:

Algorithm 3.4. Let $f_{1}: H_{1} \rightarrow H_{1}$ and $f_{2}: H_{2} \rightarrow H_{2}$ be two contractions with constants $\rho_{1}, \rho_{2} \in[0,1)$ and $\left\{t_{k}\right\} \subset[0,1]$. Let $x_{0} \in H_{1}, y_{0} \in H_{2}$ be arbitrary. Let $\left\{\alpha_{k}\right\} \subset[0,1]$ and $\left\{\beta_{k}\right\} \subset[0,1]$. Assume that the $k$-th iterate $x_{k} \in H_{1}, y_{k} \in H_{2}$ has been constructed, then we calculate $(k+1)$-th iterate $\left(x_{k+1}, y_{k+1}\right)$ via the formula

$$
\left\{\begin{array}{l}
u_{k}=x_{k}-\gamma_{k} A^{*}\left(A x_{k}-B y_{k}\right), \\
x_{k+1}=t_{k} f_{1}\left(x_{k}\right)+\left(1-t_{k}\right)\left(\alpha_{k} u_{k}+\left(1-\alpha_{k}\right) u\left(u_{k}\right)\right), \\
v_{k}=y_{k}+\gamma_{k} B^{*}\left(A x_{k}-B y_{k}\right), \\
y_{k+1}=t_{k} f_{2}\left(y_{k}\right)+\left(1-t_{k}\right)\left(\beta_{k} v_{k}+\left(1-\beta_{k}\right) T\left(v_{k}\right)\right) .
\end{array}\right.
$$

By Theorem 3.3, we obtain the following result.

Corollary 3.5. Let $\mathrm{H}_{1}, \mathrm{H}_{2}, \mathrm{H}_{3}$ be real Hilbert spaces. Given two bounded linear operators $\mathrm{A}: \mathrm{H}_{1} \rightarrow \mathrm{H}_{3}, \mathrm{~B}: \mathrm{H}_{2} \rightarrow$ $\mathrm{H}_{3}$, let $\mathrm{U}: \mathrm{H}_{1} \rightarrow \mathrm{H}_{1}$ and $\mathrm{T}: \mathrm{H}_{2} \rightarrow \mathrm{H}_{2}$ be $\tau$-demicontractive and $\mu$-demicontractive, respectively. Suppose that $\mathrm{I}-\mathrm{U}, \mathrm{I}-\mathrm{T}$ are demiclosed at origin and the solution set $\Gamma$ of the SEFP (1.2) is nonempty. Assume that the following conditions are satisfied:

(i) $\rho_{1}, \rho_{2} \in\left[0, \frac{1}{\sqrt{2}}\right)$;

(ii) $\lim _{k \rightarrow \infty} t_{k}=0$ and $\sum_{k=0}^{\infty} t_{k}=\infty$;

(iii) $\tau<\liminf _{k \rightarrow \infty} \alpha_{k} \leqslant \lim \sup _{k \rightarrow \infty} \alpha_{k}<1$;

(iv) $\mu<\liminf _{k \rightarrow \infty} \beta_{k} \leqslant \lim \sup _{k \rightarrow \infty} \beta_{k}<1$;

(v) $\gamma_{\mathrm{k}} \in\left(\varepsilon, \frac{2}{\lambda_{\mathrm{A}}+\lambda_{\mathrm{B}}}-\varepsilon\right)$,

where $\lambda_{A}, \lambda_{B}$ stand for the spectral radiuses of $A^{*} A$ and $B^{*} B$, respectively and $\varepsilon>0$ is small enough.

Then the sequence $\left\{\left(x_{k}, y_{k}\right)\right\}$ generated by Algorithm 3.4 converges strongly to a solution $\left(x^{*}, y^{*}\right)$ of the SEFP (1.2) which is the unique solution of the VIP (3.2).

If $\mu=\tau=0$, since every 0 -demicontractive mapping is quasi-nonexpansive, from Corollary 3.5 we also have the following corollary. 
Corollary 3.6. Let $\mathrm{H}_{1}, \mathrm{H}_{2}, \mathrm{H}_{3}$ be real Hilbert spaces. Given two bounded linear operators $A: \mathrm{H}_{1} \rightarrow \mathrm{H}_{3}, \mathrm{~B}: \mathrm{H}_{2} \rightarrow$ $\mathrm{H}_{3}$, let $\mathrm{U}: \mathrm{H}_{1} \rightarrow \mathrm{H}_{1}$ and $\mathrm{T}: \mathrm{H}_{2} \rightarrow \mathrm{H}_{2}(1 \leqslant j \leqslant q)$ be quasi-nonexpansive with the solution set $\Gamma$ of the SEFP (1.2) is nonempty. Suppose that $\mathrm{I}-\mathrm{U}, \mathrm{I}-\mathrm{T}$ are demiclosed at origin. Assume that the following conditions are satisfied:

(i) $\rho_{1}, \rho_{2} \in\left[0, \frac{1}{\sqrt{2}}\right)$;

(ii) $\lim _{k \rightarrow \infty} t_{k}=0$ and $\sum_{k=0}^{\infty} t_{k}=\infty$;

(iii) $0<\liminf _{k \rightarrow \infty} \alpha_{k} \leqslant \lim \sup _{k \rightarrow \infty} \alpha_{k}<1$;

(iv) $0<\liminf _{k \rightarrow \infty} \beta_{k} \leqslant \lim \sup _{k \rightarrow \infty} \beta_{k}<1$;

(v) $\gamma_{\mathrm{k}} \in\left(\varepsilon, \frac{2}{\lambda_{\mathrm{A}}+\lambda_{\mathrm{B}}}-\varepsilon\right)$,

where $\lambda_{A}, \lambda_{B}$ stand for the spectral radiuses of $A^{*} A$ and $B^{*} B$, respectively and $\varepsilon>0$ is small enough. Then the sequence $\left\{\left(x_{k}, y_{k}\right)\right\}$ generated by Algorithm 3.4 converges strongly to a solution $\left(x^{*}, y^{*}\right)$ of the SEFP (1.2) which is the unique solution of the VIP (3.2).

Remark 3.7. Theorem 3.3 extends and develops [21, Theorem 3.2] from the following aspects:

(a) Two quasi-nonexpansive mappings $\mathrm{U}$ and $\mathrm{T}$ are extended to two finite family of demicontractive mappings $\left\{U_{i}\right\}_{i=1}^{p}$ and $\left\{T_{j}\right\}_{j=1}^{q}$.

(b) The parameter sequence $\left\{\omega_{k}\right\}$ be replaced by two different parameter sequences $\left\{\alpha_{k}^{i}\right\}$ and $\left\{\beta_{k}^{j}\right\}$.

(c) The split equality fixed point problem is extended to the multiple-set split equality common fixedpoint problem.

(d) The authors did not give the proof of unique solution of the VIP (3.2) in [21], which leads to an incomplete proof. In this paper we prove it (see Step 1 in the proof). And the VIP (3.2) in this paper is also more general than that in [21].

Now first we shall give an example which satisfies all the conditions of the solution set $\Gamma$ of the MSECFP (1.4), the mappings $\left\{\mathrm{U}_{i}\right\}_{i=1}^{p}$, and $\left\{\mathrm{T}_{j}\right\}_{j=1}^{\mathrm{q}}$ in Theorem 3.3.

Example 3.8. Let $\mathrm{H}_{1}=\mathrm{H}_{2}=\mathrm{H}_{3}=\ell_{2}$ and let $i \in\{1,2, \cdots, p\}$ and $j \in\{1,2, \cdots, q\}$ be arbitrarily fixed. Let $U_{i}, T_{j}: \ell_{2} \rightarrow \ell_{2}$ be defined by $U_{i} x=-2 i x$ and $T_{j} x=-(2 j+1) x$ for all $x \in \ell_{2}$. Then it is easy to see that $\cap_{i=1}^{p} F\left(U_{i}\right)=\{0\}=\cap_{j=1}^{q} F\left(T_{j}\right)$ and $A 0=0=B 0$. Thus $\Gamma=\{(0,0)\} \neq \emptyset$. Also $U_{i}$ is $\tau_{i}$-demicontractive and $T_{j}$ is $\theta_{j}$-demicontractive, where $\tau_{i}=\frac{2 i-1}{2 i+1}$ and $\theta_{j}=\frac{j}{j+1}$, then $I-U_{i}$ and $I-T_{j}$ are demiclosed at 0 .

Indeed, for any $i \in\{1,2, \cdots, p\}$ and $j \in\{1,2, \cdots, q\}$, similar to the proof of Example 2.5 in [4], we have $U_{i}$ is $\tau_{i}$-demicontractive and $T_{j}$ is $\theta_{j}$-demicontractive. Meanwhile, $I-U_{i}$ is obviously demiclosed at 0 . For, whenever $\left\{x_{n}\right\}$ is any sequence in $\ell_{2}$ such that $x_{n} \rightarrow x \in \ell_{2}$ and $\left\|x_{n}-U_{i} x_{n}\right\| \rightarrow 0$, we readily see that $x=0 \in \mathrm{F}\left(\mathrm{U}_{\mathrm{i}}\right)$. Also, $\mathrm{I}-\mathrm{T}_{j}$ are demiclosed at 0 .

Next we give an example which satisfies the conditions (iii)-(iv) in Theorem 3.3.

Example 3.9. For $k \geqslant 0$, we can take $\alpha_{k}^{0}=\tau+\frac{1-\tau}{p+1}+\frac{1-\tau}{(p+2)(k+1)}, \alpha_{k}^{1}=\alpha_{k}^{2}=\cdots=\alpha_{k}^{p}=\frac{1-\tau}{p+1}-\frac{1-\tau}{p(p+2)(k+1)}$, $\beta_{\mathrm{k}}^{0}=\mu+\frac{1-\mu}{\mathrm{q}+1}+\frac{1-\mu}{(\mathrm{q}+2)(\mathrm{k}+1)}, \quad \beta_{\mathrm{k}}^{1}=\beta_{\mathrm{k}}^{2}=\cdots=\beta_{\mathrm{k}}^{\mathrm{q}}=\frac{1-\mu}{\mathrm{q}+1}-\frac{1-\mu}{\mathrm{q}(\mathrm{q}+2)(\mathrm{k}+1)}$.

\section{Numerical examples}

In this section, in order to demonstrate the effectiveness, realization and convergence of the algorithm of Theorem 3.3, we consider the following example in $(\mathbb{R},\|\cdot\|)$.

Example 4.1 (Numerical Example). Let $\mathrm{H}_{1}=\mathrm{H}_{2}=\mathrm{H}_{3}=\mathbb{R}$ and $\mathrm{p}=\mathrm{q}=3$. Let $\mathrm{f}_{1}, \mathrm{f}_{2}: \mathbb{R} \rightarrow \mathbb{R}$ be defined by $f_{1}(x)=f_{2}(x)=\frac{1}{2} x$. Let $A, B: \mathbb{R} \rightarrow \mathbb{R}$ be defined by $A x=B x=-x$. For any $i, j \in\{1,2,3\}$, let $U_{i}, T_{j}: \mathbb{R} \rightarrow \mathbb{R}$ be defined by $U_{i} x=-2 i x$ and $T_{j} x=-(2 j+1) x$, respectively. Let the sequence $\left\{\left(x_{k}, y_{k}\right)\right\}$ be generated iteratively by (3.1), where $\alpha_{k}^{0}=\frac{6}{7}, \alpha_{k}^{1}=\alpha_{k}^{2}=\alpha_{k}^{3}=\frac{1}{21}, \beta_{k}^{0}=\frac{7}{8}, \beta_{k}^{1}=\beta_{k}^{2}=\beta_{k}^{3}=\frac{1}{24}$ and $t_{k}=\frac{1}{k+2}$ for all $k \geqslant 0$. Then, the sequence $\left\{\left(x_{k}, y_{k}\right)\right\}$ converges strongly to $(0,0)$. 
Solution: It is easy to see that $\cap_{i=1}^{3} F\left(U_{i}\right)=\{0\}=\cap_{j=1}^{3} F\left(T_{j}\right)$ and $A 0=0=B 0$. Thus $\Gamma=\{(0,0)\} \neq \emptyset$. Also $U_{i}$ is $\tau_{i}$-demicontractive and $T_{j}$ is $\theta_{j}$-demicontractive, where $\tau_{i}=\frac{2 i-1}{2 i+1}$ and $\theta_{j}=\frac{j}{j+1}$, and $I-U_{i}$ and $I-T_{j}$ are demiclosed at $0, i, j=1,2,3$. Then $\tau=\frac{5}{7}$ and $\mu=\frac{3}{4}$. From the definition of $A$ and $B, \lambda_{A}=\lambda_{B}=1$, we choose $\gamma_{k}=\gamma=\frac{1}{2}$. It can be observed that all the assumptions of Theorem 3.3 are satisfied.

Then the scheme (3.1) can be simplified as

$$
\left\{\begin{array}{l}
x_{k+1}=\frac{2 k+9}{14(k+2)} x_{k}+\frac{k+1}{7(k+2)} y_{k \prime} \\
y_{k+1}=\frac{k+1}{8(k+2)} x_{k}+\frac{k+5}{8(k+2)} y_{k}, \quad k \geqslant 0
\end{array}\right.
$$

Utilizing the scheme (4.1), we report the numerical results in Table 1 and Table 2. In addition, Figure 1 also demonstrates Theorem 3.3.

Table 1: The values of the sequences $\left\{x_{k}\right\}$ and $\left\{y_{k}\right\}$ with initial values $x_{0}=1, y_{0}=1$.

\begin{tabular}{cccccc}
\hline$k$ & & $x_{k}$ & & \\
\hline $0-4$ & 1.000000000000 & 0.392857142857 & 0.138605442177 & 0.045728559281 & 0.014446237679 \\
$5-9$ & 0.004436210261 & 0.001336054152 & 0.000396689517 & 0.000116491267 & 0.000033907453 \\
$\vdots$ & $\vdots$ & $\vdots$ & $\vdots$ & $\vdots$ & $\vdots$ \\
$10-14$ & $9.7979 \times 10^{-6}$ & $2.8140 \times 10^{-6}$ & $8.0400 \times 10^{-7}$ & $2.2869 \times 10^{-7}$ & $6.4795 \times 10^{-8}$ \\
$\vdots$ & $\vdots$ & $\vdots$ & $\vdots$ & $\vdots$ & $\vdots$ \\
$21-25$ & $8.7888 \times 10^{-12}$ & $2.4428 \times 10^{-12}$ & $6.7796 \times 10^{-13}$ & $1.8789 \times 10^{-13}$ & $5.2006 \times 10^{-14}$ \\
$26-30$ & $1.4377 \times 10^{-14}$ & $3.9703 \times 10^{-15}$ & $1.0952 \times 10^{-16}$ & $3.0184 \times 10^{-16}$ & $8.3111 \times 10^{-17}$ \\
\hline$k$ & & & & & 0.012705562136 \\
\hline $0-4$ & 1.000000000000 & 0.375000000000 & 0.126488095238 & 0.040663531037 & 0.00029669088 \\
$5-9$ & 0.003887109325 & 0.001169434907 & 0.000347127548 & 0.000101931204 & 0.000029 \\
$\vdots$ & $\vdots$ & $\vdots$ & $\vdots$ & $\vdots$ & $\vdots$ \\
$10-14$ & $8.5732 \times 10^{-6}$ & $2.4622 \times 10^{-6}$ & $7.0350 \times 10^{-7}$ & $2.0010 \times 10^{-7}$ & $5.6696 \times 10^{-8}$ \\
$\vdots$ & $\vdots$ & $\vdots$ & $\vdots$ & $\vdots$ & $\vdots$ \\
$21-25$ & $7.6902 \times 10^{-12}$ & $2.1375 \times 10^{-12}$ & $5.9322 \times 10^{-13}$ & $1.6441 \times 10^{-13}$ & $4.5505 \times 10^{-14}$ \\
$26-30$ & $1.2580 \times 10^{-14}$ & $3.4740 \times 10^{-15}$ & $9.5834 \times 10^{-16}$ & $2.6411 \times 10^{-16}$ & $7.2723 \times 10^{-17}$ \\
\hline
\end{tabular}

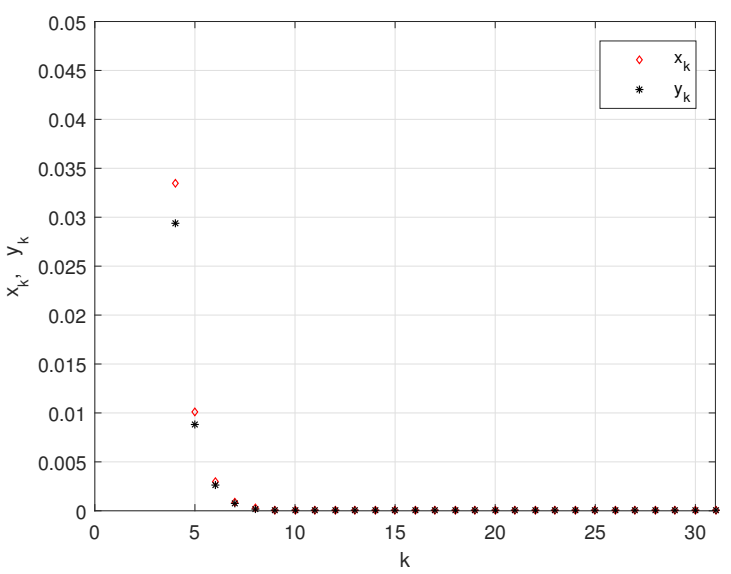

(a) Initial values: $x_{0}=1, y_{0}=1$

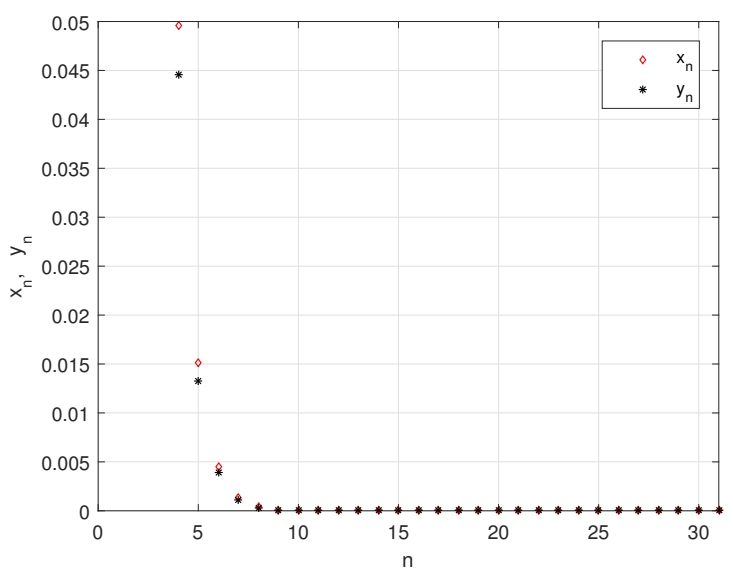

(b) Initial values: $x_{0}=1, y_{0}=2$.

Figure 1: The convergence of $\left\{x_{k}\right\}$ and $\left\{y_{k}\right\}$. 
Table 2: The values of the sequences $\left\{x_{k}\right\}$ and $\left\{y_{k}\right\}$ with initial values $x_{0}=1, y_{0}=2$.

\begin{tabular}{cccccc}
\hline$k$ & & $x_{k}$ & \\
\hline $0-4$ & 1.000000000000 & 0.464285714286 & 0.187074829932 & 0.065988672255 & 0.021408939851 \\
$5-9$ & 0.006632614002 & 0.002002531128 & 0.000594937394 & 0.000174731518 & 0.000050860910 \\
$\vdots$ & $\vdots$ & $\vdots$ & $\vdots$ & $\vdots$ & $\vdots$ \\
$10-14$ & $1.4697 \times 10^{-5}$ & $4.2210 \times 10^{-6}$ & $1.2060 \times 10^{-6}$ & $3.4303 \times 10^{-7}$ & $9.7192 \times 10^{-8}$ \\
$\vdots$ & $\vdots$ & $\vdots$ & $\vdots$ & $\vdots$ & $\vdots$ \\
$21-25$ & $1.3183 \times 10^{-11}$ & $3.6643 \times 10^{-12}$ & $1.0169 \times 10^{-12}$ & $2.8184 \times 10^{-13}$ & $7.8009 \times 10^{-14}$ \\
$26-30$ & $2.1566 \times 10^{-14}$ & $5.9554 \times 10^{-15}$ & $1.6429 \times 10^{-15}$ & $4.5277 \times 10^{-16}$ & $1.2467 \times 10^{-16}$ \\
\hline$k$ & & & & & \\
\hline $0-4$ & 2.000000000000 & 0.687500000000 & 0.210565476190 & 0.063599463223 & 0.019318759870 \\
$5-9$ & 0.005852365377 & 0.001755702460 & 0.000520788203 & 0.000152902189 & 0.000044503901 \\
$\vdots$ & $\vdots$ & $\vdots$ & $\vdots$ & $\vdots$ & $\vdots$ \\
$10-14$ & $1.2860 \times 10^{-5}$ & $3.6934 \times 10^{-6}$ & $1.0552 \times 10^{-5}$ & $3.0015 \times 10^{-7}$ & $8.5043 \times 10^{-8}$ \\
$\vdots$ & $\vdots$ & $\vdots$ & $\vdots$ & $\vdots$ & $\vdots$ \\
$21-25$ & $1.1535 \times 10^{-11}$ & $3.2062 \times 10^{-12}$ & $8.8982 \times 10^{-13}$ & $2.4661 \times 10^{-13}$ & $6.8258 \times 10^{-14}$ \\
$26-30$ & $1.8870 \times 10^{-14}$ & $5.2110 \times 10^{-15}$ & $1.4375 \times 10^{-15}$ & $3.9617 \times 10^{-16}$ & $1.0908 \times 10^{-16}$ \\
\hline
\end{tabular}

\section{Conclusion}

In this work, we study the MSECFP (1.4) which is a generalization of the SEFP (1.2). In order to obtain the strong convergence result, we introduce a new parallel algorithm combining viscosity approximation methods for the MSECFP (1.4) of demicontractive mappings in Hilbert spaces. The results we obtained mainly generalize and extend the ones in [21] from two quasi-nonexpansive mappings to two finite family of demicontractive mappings and from the SEFP (1.2) to the MSECFP (1.4). Meanwhile, we give the numerical example to demonstrate the effectiveness, realization and convergence of our algorithm. We desire that the results presented here will be useful and valuable for researchers who study the branch of split feasibility problems and related applications.

\section{Acknowledgment}

The author is extremely grateful to the referees for their useful suggestions that improved the content of the paper. This research is supported by the Natural Science Foundation of China (no. 11401388, 11671365), the Natural Science Foundation of Zhejiang (no. LQ13A010007, LY14A010006).

\section{References}

[1] C. Byrne, Iterative oblique projection onto convex sets and the split feasibility problem, Inverse Problems, 18 (2002), 441-453. 1

[2] Y. Censor, T. Elfving, A multiprojection algorithm using Bergman projections in a product space, Numer. Algorithms, 8 (1994), 221-239. 1

[3] S. S. Chang, L. Wang, Y. Zhao, On a class of split equality fixed point problems in Hilbert spaces, J. Nonlinear Var. Anal., 1 (2017), 201-212. 1

[4] C. E. Chidume, P. Ndambomve, A. N. Bello, The split equality fixed point problem for demi-contractive mappings, J. Nonlinear Anal. Optimiz., 6 (2015), 61-69. 3

[5] S. Y. Cho, B. A. Bin Dehaish, X.-L. Qin, Weak convergence of a splitting algorithm in Hilbert spaces, J. Appl. Anal. Comput., 7 (2017), 427-438. 1

[6] K. Goebel, W. A. Kirk, Topics in Metric Fixed Point Theory, Cambridge University Press, Cambridge, (1990). 2

[7] S. He, C. Yang, Solving the variational inequality problem defined on intersection of finite level sets, Abstr. Appl. Anal., 2013 (2013), 8 pages. 2.8

[8] M. C. Joshi, R. K. Bose, Some topics in nonlinear functional analysis, John Wiley and Sons, Inc., New York, (1985). 2.9 
[9] G. Marino, H.-K. Xu, Weak and strong convergence theorems for strict pseudo-contractions in Hilbert spaces, J. Math. Anal. Appl., 329 (2007), 336-346. 2.2, 2.7

[10] A. Moudafi, The split common fixed-point problem for demi-contractive mappings, Inverse Problems, 26 (2010), 587-600. 2.6

[11] A. Moudafi, E. Al-Shemas, Simultaneous iterative methods for split equality problem, Trans. Math. Program. Appl., 1 (2013), 1-11. 1, 1

[12] X.-L. Qin, S. Y. Cho, Convergence analysis of a monotone projection algorithm in reflexive Banach spaces, Acta Math. Sci. Ser. B Engl. Ed., 37 (2017), 488-502. 1

[13] D. R. Sahu, J. C. Yao, A generalized hybrid steepest descent method and applications, J. Nonlinear Var. Anal., 1 (2017), 111-126. 1

[14] W. Takahashi, Nonlinear Functional Analysis, Yokohama Publishers, Yokohama, (2000). 2

[15] H.-K. Xu, Iterative methods for the split feasibility problem in infinite-dimensional Hilbert spaces, Inverse Problems, 2010 (2010), 17 pages. 1

[16] I. Yamada, The hybrid steepest descent method for the variational inequality problem over the intersection of fixed point sets of nonexpansive mappings. Inherently Parallel Algorithm for Feasibility and Optimization and Their Applications, Stud. Comput. Math., 8 (2001), 473-504. 2.10

[17] Y.-H. Yao, R. P. Agarwal, M. Postolache, Y.-C. Liou, Algorithms with strong convergence for the split common solution of the feasibility problem and fixed point problem, Fixed Point Theory Appl., 2014 (2014), 14 pages. 1

[18] Y.-H. Yao, M. Postolache, Y.-C. Liou, Strong convergence of a self-adaptive method for the split feasibility problem, Fixed Point Theory Appl., 2013 (2013), 12 pages. 1

[19] Y.-H. Yao, J. Wu, Y.-C. Liou, Regularized methods for the split feasibility problem, Abstr. Appl. Anal., 2012 (2012), 13 pages. 1

[20] J. Zhao, S. Wang, Mixed iterative algorithms for the multiple-set split equality common fixed-point problems without prior knowledge of operator norms, Optimization, 65 (2016), 1069-1083. 1

[21] J. Zhao, S. Wang, Viscosity approximation methods for the split equality common fixed point problem of quasi-nonexpansive operators, Acta Math. Sci. Ser. B Engl. Ed., 36 (2016), 1474-1486. 1, 3.7, 5 